\title{
Ser paciente renal crónico desde la perspectiva de la enfermera: una mirada existencialista en el desvelar de la evidencia cualitativa
}

\author{
Carolina Giordani-da Silva ${ }^{1}$, Maria da Graça Oliveira-Crossetti ${ }^{1}$, Maravilla Giménez-Fernández² , Janaína \\ Prates $^{1}$ \\ ${ }^{1}$ Universidad Federal de Rio Grande do Sul. Grupo de investigación NECE. Brasil \\ 2 Universidad Católica San Antonio. Grupo de investigación "Pensamiento y lenguaje enfermero en el contexto \\ social". Murcia. España
}

Como citar este artículo:

Silva CG, Crossetti MG0, Giménez-Fernández M, Prates J.

Ser paciente renal crónico desde la perspectiva de la enfermera: una mirada existencialista en el desvelar de la evidencia cualitativa. Enferm Nefrol. 2021 Abr-Jun;24(2):139-47

\section{Resumen}

Objetivo: Este estudio pretende desvelar la evidencia cualitativa del paciente con enfermedad renal crónica a través de la percepción del equipo de enfermería nefrológica bajo una mirada existencialista.

Material y Método: Se trata de un estudio cualitativo, exploratorio-descriptivo, con análisis basado en la teoría humanista de Paterson y Zderard y de la filosofía existencialista. Los trabajos de campo se hicieron en el servicio de nefrología en el sur de Brasil y un centro de hemodiálisis en Lorca en España. La muestra fue 12 profesionales del equipo enfermería. El estudio de los datos se realizó mediante análisis de contenido, con la identificación de categorías centrales.

Resultados/Discusión: Se identificaron tres categorías centrales: ser paciente; aceptar la enfermedad y establecer vínculos. El ser paciente se desvela como un ser cuidado que requiere atención de enfermería especializada, para el tratamiento de los síntomas físicos, pero también desde una mirada atenta y acogedora de sus necesidades emocionales. La aceptación de la enferme-

\section{Correspondencia:}

Carolina Giordani da Silva

E-mail: carol.giordani@gmail.com dad por parte de éste resulta clave para ser consciente de su existencia. Para ello el profesional de Enfermería debe establecer vínculos con el ser cuidado.

Consideraciones finales: Al desvelar esta nueva realidad y la necesidad de realizar una terapia renal sustitutiva, el ser cuidado presenta sentimientos de miedo, tristeza, desesperanza, sufrimiento por la pérdida de autonomía. Sin embargo, también se perciben sentimientos positivos como: confianza, al establecer un vínculo con el equipo de enfermería, y de esperanza y alegría ante la posibilidad de un trasplante y recuperación de su autonomía.

PALABRAS CLAVE: enfermería; existencialismo; insuficiencia renal crónica; investigación cualitativa.

\section{Being a chronic kidney patient from the nursing perspective: an existentialist look at the qualitative evidence approach}

\section{Abstract}

Objective: This study aims to reveal the qualitative evidence of the patient with chronic kidney disease through the perception of the nephrology nursing team from an existential perspective. 
Material and Method: Qualitative exploratorydescriptive study, with analysis based on the Paterson and Zderard's humanist theory and the existentialist philosophy. The field work was carried out in a nephrology service in southern Brazil and a hemodialysis center in Lorca, Spain. The sample was 12 professionals from the nursing team. The study of the data was done through content analysis, with the identification of central categories.

Results/Discussion: Three central categories were identified: being patient; accept the disease and establish links. Being a patient reveals itself as a caring being that requires specialized nursing care, for the treatment of physical symptoms, but also from an attentive and welcoming view of their emotional needs. Acceptance of the disease by the patient is key to being aware of his/her existence. For this, the nursing professional must establish links with being cared for.

Final considerations: When unveiling this new reality as well as the need to perform renal replacement therapy, the person cared for presents feelings of fear, sadness, hopelessness, suffering due to the loss of autonomy. However, positive feelings such as confidence are also perceived when establishing a bond with the nursing team, and of hope and joy at the possibility of a transplant and recovery of autonomy.

KEYWORDS: nursing; existentialism; renal insufficiency chronic; qualitative research.

\section{Introducción}

La Enfermedad Renal Crónica (ERC) se caracteriza por alteraciones en la estructura o función de los riñones durante más de tres meses, además de la pérdida progresiva e irreversible de la función renal ${ }^{1}$, siendo considerada una Enfermedad Crónica No Transmisible (ECNT). Clínicamente se divide en cinco etapas, determinadas por la tasa de filtración glomerular, donde el estadio 5 representa el grado más grave de la enfermedad, requiriendo la realización de Terapia Sustitutiva Renal (TSR) para el mantenimiento de la vida ${ }^{1,2}$.

El número de pacientes con ERC ha ido aumentando paulatinamente, considerándose un problema de salud pública mundial, con una estimación de aproximadamente 850 millones de personas con enfermedad renal crónica. En los países con ingresos altos, hay
48,3 millones de hombres y 61,7 millones de mujeres, mientras que en los países de ingresos medios y bajos esta población llega a 177,4 millones de hombres y 210,1 millones de mujeres 3 . En el último censo de diálisis de 2019 realizado en Brasil, el número estimado de nuevos enfermos con ERC fue de 42.546, dando un total de 133.464 enfermos renales crónicos ${ }^{4}$. En España, los datos del Registro Español de Enfermos Renales, en 2019, registraron 86.566 personas con $\mathrm{ERC}^{5}$.

La TSR más predominante para la ERC es la hemodiálisis, con una prevalencia de hasta el $92 \%$ de los pacientes, seguida de la diálisis peritoneal y trasplante renal. Las TSR, si bien son la única posibilidad de supervivencia para los pacientes, traen consigo una serie de cambios inherentes a la dimensión física y emocional de éste, requiriendo del equipo que los cuida una mirada que contemple todas sus necesidades. Por ello, es fundamental llevar a cabo cuidados de calidad, eficaces y seguro, minimizando el riesgo para el paciente y el profesional ${ }^{6}$.

Aun así, existe una tendencia, aunque no total, de enfocar la práctica basada en la evidencia (PBE) hacia el positivismo, esto hace que se priorice la búsqueda de resolución de los signos y síntomas mediables que presenta el paciente a las acciones de salud, de hecho, muchos investigadores con dicho pensamiento siguen en la idea de que a partir del ensayo clínico se llegará a obtener la mejor evidencia. Esto provoca que se descuide las complejidades del entorno de cuidado como los sentimientos y las emociones, ya que se enfatizan los datos medibles en detrimento de las circunstancias individuales $y / 0$ singulares ${ }^{7}$, situaciones que evidencian la condición existencial del individuo.

Según el filósofo existencialista Heidegger, la condición existencial se refiere al hombre como ser singular, que se muestra en la cotidianidad con formas de expresión que pueden ser auténticas, propias y singulares 0 inauténticas, impropias e impersonales. Bajo la óptica existencial, el ser humano posee capacidad de ver, significar y apropiarse de las cosas del mundo desde su propia perspectiva ${ }^{8}$.

En el cuidado de pacientes con ERC, además de los síntomas clínicos, es común que estos experimenten sentimientos de miedo, incertidumbre, depresión, discapacidad, entre otros, ante cambios que requieren una nueva postura para vivir, lo que para muchos no es comprensible. La rebeldía, el resentimiento, la agresión, el 
aislamiento y la desesperanza también están presentes, porque es necesario renunciar a toda su historia de vida ante el diagnóstico de una enfermedad irreversible que muchos no aceptan ${ }^{9,10}$. Por otro lado, los pacientes también reconocen que el someterse al tratamiento les permite esperar un trasplante de riñón, lo que genera esperanza ${ }^{10}$. Estos sentimientos expresan la condición existencial del individuo como ser singular, y configuran la evidencia cualitativa en la práctica clínica, ya que se insertan en la dimensión estética del ser humano, que es donde uno reconoce la existencia y el desarrollo de sensaciones y percepciones, las diversas formas, modos y sentidos de la vida, el placer de ser, la existencia del otro y el cuidado como una oportunidad para ser y construir nuevas formas de vida desarrollando la creatividad para el bienestar de la persona con respeto a la dignidad humana ${ }^{11}$. Así, se queda claro cuánto es necesario rescatar la singularidad del cuidado, donde el ser humano es el foco y no la enfermedad que presenta.

La Enfermería, insertada en este contexto, es la disciplina del campo de la salud capaz de identificar la evidencia cualitativa a través del proceso de enfermería y basándose en un marco teórico, gestiona el cuidado prestado al paciente, implementando acciones terapéuticas, promoviendo y manteniendo el orden orgánico del cuerpo, el medio ambiente, la comodidad y el bienestar, manteniendo la singularidad de la persona $^{12}$. En este sentido, se cree que la teoría humanista de Paterson y Zderard ${ }^{13}$ cuando propone concretamente que las enfermeras aborden la enfermería de manera consciente y deliberada como una experiencia existencial, llega a encontrar esta forma de mirar al "ser humano" en su singularidad, reconociendo la evidencia cualitativa presente en el contexto de la atención a la persona con ERC.

La teoría humanista de Paterson y Zderad ${ }^{13}$ presupone que la ciencia de enfermería se desarrolla a partir de las experiencias vividas entre enfermeras y pacientes, y el significado de esta experiencia es el punto de partida para el establecimiento de una relación intersubjetiva. Por lo tanto, para las autoras, la atención brindada por la enfermería continúa buscando el alcance del bienestar y el ser (potencial humano), estableciendo una reunión entre personas únicas (tú y yo), guiados por un Ilamamiento (necesidad del ser cuidado) y una respuesta intencional (del ser que cuida ya que se preocupa por el ser cuidado). Es en sí misma una forma particular de diálogo humano, o diálogo vivo, entendido como una conversación entre dos o más personas, es decir, una forma de relación intersubjetiva, que se caracteriza por reunirse, relacionarse y estar presente y a partir de esto que la enfermería conciliará la razón, la sensibilidad y la subjetividad en la atención, reconociendo el sujeto como ser existencial ${ }^{14}$.

Así, este estudio tuvo como objetivo desvelar la evidencia cualitativa del paciente con ERC a través de la percepción del equipo de enfermería nefrológica bajo una mirada existencialista. Las preguntas que guiaron este estudio fueron: ¿Cómo ve al paciente con ERC? y ¿qué cree que es más difícil para el paciente en este contexto de ERC?

\section{Material y Metodo}

Se trata de un estudio cualitativo, exploratorio-descriptivo, con un análisis basado en el marco teórico de la teoría humanista de Paterson y Zderard ${ }^{13}$ y de la filosofía existencialista, vinculado a un proyecto de investigación más amplio, donde es fundamental la mirada del equipo de enfermería sobre el ser paciente $^{14,15}$. Además, se destaca la reflexión de la investigadora sobre el tema, ya que vive el mundo de la enfermedad renal crónica.

El ámbito del estudio fueron unidades de hemodiálisis pertenecientes al Hospital de Clínicas de Porto Alegre, hospital universitario de la Universidad Federal de Rio Grande do Sul, con atención múltiple, enfocado a la educación, la investigación y la atención de la salud; junto con Servicio de Nefrología, integrado por la unidad de hemodiálisis, que atiende a pacientes con insuficiencia renal aguda o crónica de diferentes áreas del hospital, que requieren TSR, y además, en un centro de hemodiálisis en Lorca - Región de Murcia en España. La elección de desarrollar el estudio se debe al acercamiento de las investigadoras en esta área, a través de la especialización latum sensu y el desarrollo de una tesis doctoral en este campo.

La población de estudio estuvo compuesta por profesionales del equipo de enfermería y la muestra se estableció por conveniencia, mediante invitación directa a los informantes. Ésta estuvo conformada por 12 profesionales y el tamaño muestral no estaba preestablecido, es decir, no se buscaba la representatividad estadística sino la teórica, de manera que cuando se alcanzó la saturación de datos se detuvo la recogida de los mismos. Los criterios de inclusión fueron profesionales del equipo enfermería con uno o más 
años de experiencia en la institución donde trabajan, en los diferentes turnos de atención, que realizaron sus actividades durante el período de recolección de datos. Los criterios de exclusión aplicables fueron no proporcionar asistencia directa a los pacientes durante el período de investigación.

Los fundamentos bioéticos se cumplieron en base a los estándares que regulan los proyectos de investigación con seres humanos establecidos en la Resolución 466/2012, del Consejo Nacional de Salud ${ }^{16}$. El proyecto fue aprobado por el Comité de Investigación (COMPESQ) de la Facultad de Enfermería y por el Grupo de Investigación y Postgrado (GPPG) del Hospital de Clínicas de Porto Alegre con el número CAAE 23534719.7.3001.5327.

Los datos fueron recogidos entre enero y junio de 2020, a través de entrevistas semiestructuradas ${ }^{14}$ realizadas de manera individual, 3 presencialmente en un lugar privado, preservando la confidencialidad de la información y de los participantes, y 9 virtualmente, a través del programa Zoom. Las entrevistas fueron semiestructuradas y en profundidad ${ }^{14}$, caracterizada por preguntas amplias, abiertas y no evaluativas, estimulando el surgimiento de declaraciones e historias inesperadas, en las que el entrevistador manifiesta interés por ahondar en temas determinados, y tuvieron una duración promedio de 1 hora. De acuerdo con la estructura adoptada, este tipo de entrevista permite examinar en detalle los discursos de un entrevistado con experiencias relevantes, no necesitando una prueba piloto para validar las preguntas de la entrevista, ya que permite al investigador ahondar en los enunciados que aparecen durante la entrevista y que son relevantes para enriquecer el tema estudiado. También se recolectaron datos para caracterizar la muestra como edad, tiempo como profesional y tiempo de años trabajando en la institución. Las entrevistas fueron grabadas con autorización de los informantes.

Después de cada entrevista, se transcribió y se realizó una lectura exhaustiva del contenido, y a partir de las declaraciones de los participantes surgieron las unidades de análisis ${ }^{17}$, posibilitando la identificación de ideas centrales y conceptos comunes, los cuales fueron agrupados en categorías, discutidos a la luz de la teoría humanista de Paterson y Zderad ${ }^{13}$ desde la perspectiva de otros autores y la experiencia de los investigadores con filosofía existencialista en la dirección de desvelar la evidencia cualitativa presente en el mundo de la ERC.

\section{Resultados y discusión}

De los 12 participantes, 8 son enfermeras, 1 enfermero y 3 técnicos de enfermería, predominantemente mujeres $83,3 \%$ (10), con edades entre 23 y 59 años y tiempo de profesión entre 1 y 30 años, de acuerdo al Tabla 1 de caracterización de la muestra.

Para ilustrar la presentación de los resultados, se seleccionaron algunos extractos de los discursos de los profesionales de enfermería y se utilizó la siguiente estandarización: los paréntesis con puntos suspensivos [...] significan supresión en los discursos del investigador; ser que cuida - equipo de enfermería; ser cuidado-paciente. Cabe señalar que fue posible identificar en los testimonios de los participantes del estudio, tres categorías que se presentan a continuación: 1) ser paciente; 2 ) aceptando la enfermedad y 3 ) estableciendo vínculos. Estas categorías se derivaron de los relatos de los profesionales sobre su experiencia existencial en este contexto de ERC y TSR, en los que exponían sus percepciones sobre el paciente con ERC. 


\section{Ser paciente}

"Ser cuidado" - paciente es un "estar en el mundo de la ERC". La concibe como una existencia compleja, con muchas especificidades. El conocimiento del diagnóstico de enfermedad hace que el ser cuidado establezca una relación de preocupación con este mundo, que necesita ser desvelada ya que dificulta vislumbrar su potencial existencial. Por eso se convierte en un desafío para el ser cuidador, como refieren algunos participantes del estudio:

"... es un desafío diario, porque en realidad son pacientes que demandan mucha atención, demandan mucho cuidado, no solo psicológico [...], de conocimiento, porque el paciente con ERC tiene otras enfermedades también, a veces con diabetes e hipertensión, entonces es un desafío, porque tenemos que estar siempre aprendiendo y atentos". (ENF1)

"... el paciente renal crónico, es diferente para nosotros, tiene especificidades de la enfermedad, entonces a veces tenemos ese paciente que es más frágil, incluso aceptando la enfermedad también, ya que hay ese paciente que acepta mejor y el tratamiento no es tan complicado". (TENF3)

Se entiende que la importancia de la Teoría de Enfermería Humanista aplicada en el contexto de la Nefrología, con el paciente renal crónico, radica en el hecho de perfeccionar y orientar en el cuidado al ser que cuida existencialmente, ya que esta teoría se basa en el encuentro y el diálogo auténtico entre el ser enfermera y el ser paciente ${ }^{18}$.

Se desvela este ser cuidado como un ser humano que requiere cuidados de enfermería especializados, desde el tratamiento de los síntomas físicos y la atención a las limitaciones funcionales, los trastornos mentales y las necesidades educativas, pero también desde una mirada atenta y acogedora de sus necesidades emocionales.

En este sentido, la calidad del servicio brindado está asociada al rol del ser que cuida, con conocimiento especializado y atento a la interacción, ser cuidado - ser que cuida, predictores importantes que inciden en la asistencia prestada ${ }^{19}$. Esto requiere una actualización constante por parte de los seres que cuidan, como apunta un participante del estudio:

"... el paciente cambia de perfil y hay que actualizarse, siempre hay que estudiar, [...], buscando cosas nuevas. Entonces para mí es un desafío diario, y también tenemos que mirar más allá del horizonte. La enfermera Nefrológica [...], puede ver más allá del horizonte y ver nuevas alternativas de cuidado para este paciente [...] porque cambia el perfil y hay que estar actualizado". (ENF4)

Sin embargo, el conocimiento debe venir junto con la presencia auténtica en cada encuentro de cuidado, que permita establecer una relación intersubjetiva, es decir, sujeto-sujeto, y así percibir a cada ser cuidado en su singularidad, de ahí la importancia de identificar la evidencia cualitativa presente en este contexto de ERC ${ }^{13,20}$ y así, establecer nuevas alternativas de cuidado a estos pacientes.

El ser que cuida describe al ser cuidado en este mundo de la ERC, como un ser que presenta miedo, tristeza, desesperanza, momentos de rabia, dolor y sufrimiento por la pérdida de autonomía e inseguridad por vivir constantemente la angustia por la cercanía a la muerte, pero también que tienen alegría y esperanza cuando pueden optar por una TSR que les aporta más autonomía, como la diálisis peritoneal y el trasplante, desvelándose como pacientes que tienen inestabilidades emocionales, requiriendo la mirada atenta de enfermería.

"Percibo sentimientos de tristeza, tienen muchos síntomas depresivos, de perder en parte su autonomía, su poder adquisitivo [...] un sentimiento de impotencia, de tener la enfermedad, de tener que adaptarse y hacer todo lo posible para vivir con eso [...], a veces soledad, desesperanza, es sufrimiento por las limitaciones que trae la enfermedad. Es un paciente que vive muy cerca de la muerte, entonces muchos relatan que tienen miedo a morir". (ENF2)

"Tiene ese proceso de negación, después de no aceptar, que se rebela, entonces come, bebe, hace de todo, y luego llega un punto, que realmente quiere cuidarse [...], porque no quieren volver a la máquina, porque en realidad la máquina es una prisión". (ENF6)

Los cambios en la vida de los seres cuidados debido a ERC traen consigo sentimientos de resentimiento, agresión, violencia y aislamiento, que eventualmente se trasladan a los seres que cuidan o incluso repercuten en una actitud de inconformismo con su situación, llevándolos a la no adherencia al tratamiento ${ }^{21,22}$, como aporta esta enfermera:

"Proyectan en el profesional [...] como si sus problemas, como si tuviéramos alguna culpa, responsabilidad, [...]. Proyectan sus frustraciones en nosotros". (ENF3)

De esta forma queda claro que, los sentimientos percibidos por el ser que cuida están en línea con los hallazgos 
de otros estudios que señalan que, en el transcurso de las sesiones de hemodiálisis, el ser cuidado sufre alteraciones fisiológicas como cansancio, malestar, caída de la presión arterial y calambres, y también cursan con agotamiento emocional, que se traduce en sentimientos de tristeza, rebeldía, inseguridad, frustración y preocupación por el futuro ${ }^{20,23}$. Sin embargo, el trasplante de riñón, aunque es una TSR se ve por el ser cuidado como una posibilidad para seguir proyectándose, superándose a sí mismo y a la enfermedad, trascendiendo hacia su libertad ${ }^{8}$. Así, en el mundo de la ERC también es posible revelar sentimientos positivos, como la esperanza de realizar un trasplante y la alegría de mejorar la calidad de vida recuperando parte de la autonomía, como ha dicho una enfermera:

"... a veces les entusiasma la posibilidad de poder hacer diálisis en casa cuando cambian el método de tratamiento. A veces sentimientos de alegría porque lograron entrar en la lista de trasplantes, esto es algo que motiva, [...]. Como te dije, tienen altibajos". (ENF1)

\section{Aceptando la Enfermedad}

El ser cuidado vive un proceso de enfermedad grave e incierta que representa lo desconocido, dando lugar a diferentes sentimientos que muchas veces dificultan el reconocerse como persona enferma y la adaptación al tratamiento. Éste requiere una estricta rutina con los alimentos y la TSR elegida, además de dar lugar a periodos de hospitalización, lo que le conlleva a la separación de los miembros de su familia.

En este sentido, el ser que cuida se da cuenta de que las restricciones que impone la ERC, destacando las restricciones dietéticas, el aislamiento social y la pérdida de autonomía, son las principales barreras para que el ser cuidado acepte la enfermedad y se adhiera al tratamiento.

"Creo que lo más difícil para el paciente es el tema de la limitación que pasa de un día para otro. Entonces, tomando la libertad que tenía ahí fuera, parece que se siente atrapado en ese contexto". (ENF5)

"Pues creo que lo más difícil para ellos es la diálisis 3 veces por semana, la dependencia de la máquina de diálisis y el tiempo de tratamiento". (ENF7)

"Creo que lo más difícil para ellos es reestructurar su vida, es aceptar que están en diálisis, que es un tratamiento para siempre y que tienen que adaptar su vida a la rutina del tratamiento". (ENF9)

El descubrimiento del diagnóstico, que trae consigo la condición de estar enfermo, es siempre una limitación más o menos grave de las posibilidades de relación que el ser cuidado puede mantener en el mundo y, por tanto, de su libertad ${ }^{24}$. Así, la TSR, si bien es la única forma de sobrevivir, es entendida como limitante, ya que provoca cambios en la rutina diaria, generando un impacto en el estilo de vida, principalmente por la dependencia de una máquina, en el caso de la hemodiálisis, o cambios diarios continuos en la diálisis peritoneal, lo que en general dificulta la aceptación de la enfermedad y la adherencia a la terapia propuesta ${ }^{21,25-26}$.

En este sentido, la comprensión es uno de los rasgos ontológico-existenciales de los seres, es importante que el ser que cuida, oriente de manera clara y objetiva al ser cuidado sobre su diagnóstico, las modalidades de TSR disponibles y los cuidados que cada uno de ellos demanda, posibilitando elegir el tratamiento donde el ser cuidado puede proyectarse en el mundo con menos sufrimiento y restricciones, contribuyendo positivamente a la aceptación y afrontamiento de la enfermedad $^{26}$. Se sabe que los seres cuidados describen la experiencia de la hemodiálisis como una lucha diaria por aceptar la enfermedad y adaptarse al tratamiento, el cual es responsable de una vida de restricciones, imponiendo limitaciones que afectan los aspectos biológicos, psicológicos y sociales de su vida, conduciendo a una ruptura en su estilo de vida ${ }^{19,27}$. Ante esto, es necesario que el ser cuidado tenga siempre la presencia de alguien que le acoja, apoye y consuele, creando una relación de confianza entre ser cuidado y ser que cuida $^{18}$.

\section{Estableciendo Vínculos}

El establecimiento del vínculo se crea a partir de una relación de confianza entre YO-TÚ y YO-ESO, donde se impregna todo el proceso de cuidar, bien por el llamamiento del ser cuidado o ante situaciones en las que el ser que cuida lo considere importante, facilitando el proceso de aceptación de esta nueva condición de ser y estar en el mundo de la ERC del ser cuidado. La presencia y el encuentro también permean la relación YO-NOSOTROS, es decir, la relación de cuidado entre ser cuidado y ser que cuida. Dicho cuidado se caracteriza, en la Teoría de la Enfermería Humanística, como una mediación que se da entre sus pares, es decir, pacientes, familiares y profesionales de la salud, especialmente enfermería. Así, tanto la relación YOTÚ como la YO-NOSOTROS están representadas por el compromiso de todos los cuidadores de brindar un verdadero encuentro terapéutico con miras al bienestar del ser cuidado ${ }^{18}$. 
En este sentido, durante las diferentes TSR hacen que el ser que cuida se convierta en una presencia constante en la vida del ser cuidado, que por su soledad busca suplir esta carencia con el equipo, lo que establece fuertes lazos entre el ser que cuida y el ser que es cuidado, según los siguientes relatos:

"Yo puedo ayudar y acompañar al paciente desde el principio, tú puedes estar ahí sumando a su vida [...] ellos piensan que incluso eres parte de la familia. Y tú también te haces amigo, porque ellos tienen confianza en $t^{\prime \prime}$. (TENF3)

"Terminas conociendo bien al paciente, tienes un factor que facilita tu vida diaria con él, pasas 4 horas, 3 veces a la semana con el mismo paciente, terminas conociendo, tanto la parte clínica, en cuanto a la parte física y social del paciente, conoces bien sus necesidades, puedes darle continuidad, ver si estás recibiendo respuestas a tus cuidados, si necesitas cambiar algo". (ENF4)

"Después de tantos años tomo el centro como si fuera mi segunda residencia, porque llevo muchos años trabajando y el trato con el paciente es de confianza, [...] lo que a veces es difícil porque, así como te tratan bien, también te tratan mal, y yo sigo aprendiendo y conociéndolos". (ENF8)

En este sentido, en Enfermería Humanística, el ser que cuida debe estar dispuesto a "estar-con" y "estar-ahí" para el ser cuidado sin posibilidades terapéuticas de curación, estableciendo vínculos y redes de apoyo que permitan apreciar adecuadamente la situación vivida al ser cuidado, para crear alternativas para su bienestar y desarrollar su estar mejor, ayudándoles a encontrar oportunidades para seguir existiendo en este mundo de $\mathrm{ERC}^{8,13,18 \text {. }}$

Queda claro entonces, que el ser que cuida "representa" una familia para el ser cuidado, porque es con ellos con quienes conviven buena parte de la semana, aumentando este vínculo y transformando esta atención ofrecida por los profesionales como si fueran realmente miembros de la familia. Este vínculo formado entre ser cuidado y ser que cuida permite la construcción de conocimiento sobre la enfermedad y el tratamiento, proporcionando una relación YO-NOSOTROS de aprendizaje continuo, potenciando la aceptación terapéutica9,18,22,26,28-29.

Por tanto, queda clara la importancia del vínculo entre los seres en el mundo de la ERC para establecer una au- téntica relación de cuidado. Para ello, el ser que cuida debe prestar asistencia con conocimientos científicos. Y a través de la acogida, la relación interpersonal, la escucha activa y el diálogo efectivo establecer una relación de confianza entre seres en el mundo de la ERC procurando el tratamiento exitoso. La confianza permite al ser cuidado explicitar sus angustias, miedos y ansiedades, y a partir de esto, el ser que cuida aprende las dificultades encontradas para, en conjunto, desarrollar estrategias que faciliten la adherencia del ser cuidado a TSR elegida9,19,22,30.

En cuanto a las limitaciones, este estudio tuvo la mirada única del ser que cuida sobre las evidencias cualitativas en el contexto del cuidado de la ERC. Se cree que deben desarrollarse nuevos estudios bajo nuevas perspectivas como la del ser cuidado y su familia, para tener un mejor conocimiento de esta experiencia en el mundo de la ERC, enriqueciendo el cuidado de enfermería. Se espera que este estudio pueda contribuir a la práctica clínica de la enfermería, así como a la docencia, en el sentido de prestar atención a la presencia de las evidencias cualitativas en el contexto del cuidado de la ERC, ante el impacto que provocan en la adaptación del ser cuidado al tratamiento $y$, en consecuencia, en la calidad de vida de los mismos.

\section{Consideraciones finales}

Este estudio buscó identificar la evidencia cualitativa presente del ser cuidado en el contexto del cuidado de la ERC desde la perspectiva del ser que cuida. En este sentido, se reconoce que, al desvelar esta nueva realidad al ser cuidado y la necesidad de someterse a TRS, aflora en éste sentimientos de miedo, tristeza, desesperanza, desamparo, soledad, sufrimiento por pérdida de autonomía, enfado, agresividad, luto, rebeldía, inconformidad con la situación vivida, frustración, así como una constante inseguridad y angustia por la proximidad a la muerte. Sin embargo, también se perciben sentimientos positivos como: confianza, al establecer un vínculo con el equipo de enfermería, y de esperanza y alegría ante la posibilidad de realizar un trasplante y recuperar parte de su autonomía. Por ello es necesario que el ser que cuida tenga una postura acogedora, de respeto, de escucha atenta, empatía y paciencia para implementar estrategias de cuidado a través de acciones educativas, que ayuden al ser cuidado a vislumbrar nuevas posibilidades de seguir existiendo y de proyectarse en el mundo, contribuyendo así a una mejor adherencia y afrontamiento de los retos inherentes a cada TSR. 
"Este trabajo se realizó con el apoyo de la Coordinación de Perfeccionamiento del Personal de Educación Superior - Brasil (CAPES) - Código de Financiamiento CAPES/PRINT - Edital 41/2-2017".

Recepción: 12-11-20

Aceptación: 02-04-21

Publicación: 30-06-21

\section{Bibliografía}

1. Daugirdas JT, Blake PG, Ing TS. Manual de diálise. 13th ed. Rio de Janeiro: Guanabara Koogan; 2016.

2. Spigolon DN, Teston EF, Souza F0, Santos B, Souza RR, Moreira Neto A. Nursing diagnoses of patients with kidney disease undergoing hemodialysis: a cross-sectional study. Rev Bras Enferm. 2018;71(4):2014-20.

3. Mills KT, Xu Y, Zhang W, Bundy JD, Chen C, Kelly $\mathrm{TN}$ et al. A systematic analysis of world-wide population-based data on the global burden of chronic kidney disease in 2010. Kidney International. 2015;88(5):950-7.

4. Neves PDMM, Sesso RCC, Thomé FS, Lugon JR, Nascimento MM. Censo Brasileiro de Diálise: análise de dados da década 2009-2018. J. Bras. Nefrol. 2020;42(2):191-200.

5. REER - Registro Español de Enfermos Renales. Informe de diálisis y Trasplante 2019. [consultado 22 feb 2020]. Disponible en: senefro.org/contents/ webstructure/INFORME_REER_SEN_2020_ WEB_SEN.pdf.

6. Danski MTR, Oliveira GLR, Pedrolo E, Lind J, Johann DA.Importância da prática baseada em evidências nos processos de trabalho do enfermeiro. Ciência, Cuidado E Saúde. 2017 abr-jun;16(2).

7. Ou CHK, Hall WA, Thorne SE. Can nursing epistemology embrace p-values? Nurs Philos. 2017; 18:e12173.
8. Heidegger M. Ser e tempo. $3^{\text {a }}$ ed. Petrópolis: Vozes; 2008.

9. Salimena AMO, Costa YCN, Amorim TV, Souza RCM. Sentimentos da pessoa em hemodiálise: percepção da equipe de enfermagem. Revista de Enfermagem do Centro-Oeste Mineiro. 2018;8:e2578.

10. Silva AS, Silveira RS, Fernandes GFM, Lunardi VL, Backes VMS. Percepções e mudanças na qualidade de vida de pacientes submetidos à hemodiálise. Rev Bras Enferm. 2011;64(5):839-44.

11. Rodríguez $S$, Cárdenas $M$, Pacheco $A L$, Ramírez $\mathrm{M}$, Ferro N, Alvarado E. Reflexión teórica sobre el arte del cuidado. Enfermería Universitária 2017;14(3):191-8.

12. Ferreira MA. 0 clássico e 0 emergente: desafios da produção, da divulgação e da utilização do conhecimento da enfermagem. Rev Bras. Enferm. 2013;66(esp):45-60.

13. Paterson JE, Zderad LT. Enfermería humanística. Ciudad de México (MEX): Editoral Limusa S.A; 1979.

14. Polit DF, BECK CT. Fundamentos de pesquisa em enfermagem: avaliação de evidências para a prática da enfermagem. $7^{\text {a }}$ edição. Porto Alegre: Artmed; 2011.

15. $0^{\prime}$ Brien BC, Harris IB, Beckman TJ, Reed DA, Cook DA. Standards for Reporting Qualitative Research: A Synthesis of Recommendations. Acad Med. 2014;89:1245-51.

16. Brasil. Resolução $n^{\circ} 466$, de 12 de dezembro de 2012. Dispõe sobre diretrizes e normas regulamentadoras de pesquisas envolvendo seres humanos. Diário Oficial da República Federativa do Brasil, Brasília, DF, 13 jun. 2013. [consultado 20 feb 2020]. Disponible en: http://bit.ly/1mTMIS3.

17. Bardin L. Análise de conteúdo. São Paulo: Edições 70; 2011.

18. França JRFS, Costa SFG, Lopes MEL, Nóbrega $M M L$, Batista PSS, Oliveira RC. Existential experience of children with cancer under palliative care. Rev Bras Enferm. 2018;71(Suppl 3):S13207. 
19. Stavropoulou A, Grammatikopoulou MG, Rovithis M, Kyriakidi K, Pylarinou A, Markaki AG. Through the Patients' Eyes: The Experience of End-Stage Renal Disease Patients Concerning the Provided Nursing Care. Healthcare. 2017;5(36).

20. Shahdadi H, Rahnama M. Experience of Nurses in Hemodialysis Care: A Phenomenological Study. J. Clin. Med. 2018;7(30).

21. Salimena AMO, Souza MO, Melo MCSC, Ferreira MR. 0 cotidiano da mulher em hemodiálise. Rev Fund Care Online. 2016 jul/set;8(3):4636-43.

22. Maciel CG, Ferraz RN, França VV, Frazão IS, Borba AKOT. Adesão ao tratamento hemodialítico: percepção dos pacientes renais crônicos. Cogitare Enferm. $2015 \mathrm{jul} / \mathrm{set} ; 20(3): 540-7$.

23. Ferreira LF, Agra G, Formiga N. Experiências e sentimentos de pacientes em terapida hemodialítica. RSC online, 2017;6(1):39-56.

24. Santos DG, Sá RN. A Existência como "Cuidado": Elaborações Fenomenológicas sobre a Psicoterapia na Contemporaneidade. Revista da Abordagem Gestáltica - Phenomenological Studies -. 2013; XIX(1):53-9.

25. Alves LO, Guedes CCP, Costa BG. Nurses actions for chronic renal patients: reflection of comprehensive care focus. J. res.: Fundam. Care. Online. 2016 jan./ mar;8(1):3907-21.
26. Ramírez-Perdomo CA. Afrontar el tratamiento de la enfermedad renal crónica. Enferm Nefrol. 2019 oct-dic;22(4):379-87.

27. Silva SM, Braido NF, Ottaviani AC, Gesualdo GD, Zazzetta MS, Orlandi FS. Social support of adults and elderly with chronic kidney disease on dialysis. Rev. Latino-Am. Enfermagem. 2016;24:e2752.

28. Pereira BS, Fernandes NS, Melo NP, Abrita $R$, Grincenkov FRS, Fernandes NMS. Beyond quality of life: a cross sectional study on the mental health of patients with chronic kidney disease undergoing dialysis and their caregivers. Health and Quality of Life Outcomes. 2017;15:74.

29. Nobahar M, Tamadon MR. Barriers to and facilitators of care for hemodialysis patients; a qualitative study. J Renal Inj Prev. 2016;5(1):39-44.

30. Delmas $P, 0^{\prime}$ Reilly L, Cara C, Brousseau S, Weidmann J, Roulet-Schwab $D$ et al. Effects on nurses quality of working life and on patients' quality of life of an educational intervention to strengthen humanistic practice among hemodialysis nurses in Switzerland: a protocol for a mixed-methods cluster randomized controlled trial. BMC Nursing. 2018;17:47.

Este artículo se distribuye bajo una Licencia Creative Commons Atribución-NoComercial 4.0 Internacional. https://creativecommons.org/licenses/by-nc/4.0/ 